\title{
Analysis of Traumatic Injury in 886 Permanent Anterior Teeth
}

\author{
Lihong Ge ${ }^{1)}$, Jie Chen ${ }^{1)}$, Yuming Zhao' ${ }^{1)}$, Bin Xia ${ }^{1)}$, Mitsutaka Kimura ${ }^{2)}$ \\ ${ }^{1)}$ Department of Pediatric Dentistry, Peking University School and hospital of Stomatology, Beijing, PR China \\ ${ }^{2}$ Department of Pediatric Dentistry, Kyushu Dental College, Kyushu, Japan
}

\begin{abstract}
Clinical data of 886 traumatized permanent anterior teeth in 512 children who were treated at the department of Pediatric Dentistry, Peking University School of Stomatology were collected. The type, reason, and mode of the trauma, the number of teeth involved in each person and time elapsed before treatment were analyzed. The results suggested that boys were more susceptible to traumatic injury of anterior teeth, and the highest prevalence of dental trauma was determined in the 8-10 years age group. Falls was the most common reason. Coronal fracture without pulp exposure was the most common type. Most children went to hospital in 2 to 24 hours after injury.
\end{abstract}

Key words: young permanent teeth, anterior teeth, tooth injuries

\section{Introduction}

The prevalence of dental trauma among children and adolescents has increased recently. Though many papers have been published abroad on the epidemiology of traumatic injury of anterior teeth, only few were about traumatic injury of young permanent anterior teeth in the population of 6 to 15 years old, none have been published in China. Data of 512 children aged form 6 to 15 with dental trauma of permanent anterior teeth treated at the department of Pediatric Dentistry, Peking University School and hospital of Stomatology in recent 5 years were investigated retrospectively in this study. The reason of injury, time elapsed after injury, proportion of different types of injury, the relationship of dental injury of anterior teeth and age, gender, and root development were analyzed to prevent dental injury of anterior teeth, protecting young permanent teeth and promoting their normal development.

\section{Materials and Methods}

Materials

512 children with dental trauma of permanent anterior teeth treated in recent 5 years at the Department of Pediatric Dentistry, Peking University School and hospital of Stomatology were involved in this study. Totally 886 teeth were injured, all of which were young permanent teeth. Clinical Data of each patient were intact. The age of the patients ranged from 6 to 15.339 children were boys and 173 were girls.

\section{Methods}

1. Dental trauma was classified according to criteria developed by Department of Pediatric Dentistry, Peking University School of Stomatology. The criteria are as follows:

(1)Concussion

(2)Tooth fracture: (1) Crown fracture: enamel fracture; enamel fracture with dentin exposure and dentin fracture; crown fracture with pulp exposure; (2) Crown root fracture; (3)Root fracture: root fracture at cervical third; root fracture at middle third; root fracture at apical third.

(3)Tooth displacement: (1) Intrusion; (2) Lateral luxation; (3) Extrusive Luxation; (4)Avulsion.

In our study, 2 and more teeth suffered different types of dental injury in one child were diagnosed as complex injury.

2. Periapical radiographs were taken to each injured tooth, and reviewed by 3 senior dentists for making a diagnosis and assessing root development.

3. Clinical data including: age, gender, reason of injury, place and time of injury, soft tissue injury, type of injuries and tooth position were recorded.

4. Proportion of different types of injury and the relationship of dental injury and age, gender, and root development were analyzed. The reason, place and time of injury, tooth position and time elapsed after injury were analyzed.

\section{Results}

1. The relationship between the type of the injury and age and gender showed that among 512 patients, 339 were boys and 173 were girls, the boy-to-girl ratio was 1.96:1. The highest frequency of trauma occurred at age 9 to 10, and the incidence of dental trauma was $18.95 \%$ and $18.76 \%$ respectively. 8-year old group was next prone to dental injury, which incidence was $15.23 \%$.

2. Time elapsed after injury: Most of the patients (41.21\%) went to hospital 2 to 24 hours after injury. In the next place, 31.64\% of the patients went to hospital after 1 to 7 days (Table 1 ).

3. Proportion of different types of dental injury and status of root development were shown in Table 2. The most common type of injury was crown fracture without pulp exposure, composing $37.3 \%$ of total injuries. $33.9 \%$ of total injuries were crown fracture with pulp exposure. The ratio of root underdeveloped-to-developed was 1.35:1.

4. Reason of injury: Falls (64.46\%) was the most common reason of dental injury, followed by accident (9.37\%) and collision (6.25). $1.95 \%$ of total injuries were caused by other reasons, and the reasons of $12.77 \%$ injuries were unknown.

5. Place of injury: The places of injuries were classified as indoors and outdoors, and the places of 178 cases (34.77\%) were unknown. $51.17 \%$ of the injuries occurred outdoors including school (23.83\%), street (22.48\%), and park. $12.11 \%$ injuries occurred indoors including school (7.03\%) and home.

6. $30.66 \%$ of the 512 patients suffered soft tissue injuries, while most of the patient (69.74\%) didn't have soft tissue injuries.

7. The distribution of tooth number and position in patients showed that the most frequently affected teeth were maxillary anterior teeth (97.07\% of total injuries), and $1.17 \%$ injuries occurred on mandibular anterior teeth. $1.76 \%$ patient had both maxillary and mandibular teeth injured. Most patients had only one central incisor injured, both central incisors injured was in the next place.

\section{Discussion}

1. In this study, 339 of the 512 children with dental trauma were boys and 173 were girls, the boy-to-girl ratio was 1.96:1, which is consistent with the ratio of 1.76:1 reported by Hunter ${ }^{1,2)}$. The 
International symposium of Maxillofacial \& Oral Regenerative Biology in Okayama 2005

results suggested that boys were susceptible to traumatic injury of anterior teeth.

2. The largest percentage of injuries was demonstrated by 10year-old schoolchildren, which was $18.95 \%$, similarly, $18.75 \%$ injuries occurred in 9-year-old group, followed by 8-year-old group (15.23\%). The highest prevalence of dental trauma was determined in the 8-10years age group for both boys and girls, which was younger than 10-12 years olds reported by Llarena ${ }^{3)}$. In Beijing, 8-10 year old children are about 3 to 5 grades in primary schools. It's necessary to wear mouthguard at this age.

3. Most of the patients went to hospital 2 to 24 hours after injury, composing $41.21 \%$ of total injuries. $5.08 \%$ of the patients went to hospital within 30 minutes, and $13.48 \%$ after 30 minutes to 2 hours. Therefore, $59.77 \%$ of the patients went to hospital within 24 hours, which is similar to $61.00 \%$ reported by Onetto ${ }^{4}$. The results suggested that most patients were treated in time, especially for patients went to hospital within 30 minutes and 2 hours. Despite types of injuries, treatment was more likely to be successful in cases treated within 24 hours. Therefore, it is essential to enhance oral health instruction, ensure the understanding of the importance of treating in time after injury by the parents, children and primary medical carer. Patients should be treated within 2 hours after injury.

4. The most common type of injury was crown fracture without pulp exposure, composing $37.3 \%$ of total injuries, which was similar to $37.90 \%$ reported by Motohiro ${ }^{5}$. Next was crown fracture with pulp exposure, the proportion of which was 33.9\%. No difference of patient distribution was observed in other trauma types.

5. Root development was normally incomplete in 6-15-year-old children. The ratio of root underdeveloped-to-developed was $1.35: 1$, except for patients with root fracture, which ratio of root underdeveloped-to-developed was 1:2. The root of young permanent teeth is short and bulky, the apical orifice is wide, and periroot tissue is floppy which renders the root resistent to fracture, while the situation of developed teeth is opposite. The success of treatment was directly related to the type of injury and root development, which should be further analyzed.

6. Among all the reasons, falls (64.46\%) was the most common one. Falls happened while playing sports, chasing each other, walking unguardedly, walking along swimming pool, and slipping under raining or snowing. Most injuries occurred outdoors (51.17\%), which is similar to previous reports ${ }^{4,6,7)}$. Dental trauma was more likely to happen on the way school and home. Most indoor injuries happened at school. Dentists should emphasize the harm of dental trauma to children, and improving the knowledge of self-protection at school.

7. The most frequently affected teeth were maxillary anterior teeth (97.07\%), 54.49\% of which had only one tooth involved, $31.45 \%$ had both central incisors injured, which is similar to previous published data ${ }^{1,4,6)}$. Maxillary central incisors are important for appearance, pronunciation and occlusion. It is the dentist's duty to take action to protect these teeth.

\section{References}

1. Castro JC, Poi WR, Manfrin TM et al. Dent Traumatol 21: 121-126; 2005.

2. Hunter ML, Hunter B, Kingdon A, et al. Endod Dent Traumatol 6: 260-264; 1990.

3. Llarena del Rosario ME, Acosta Aofaro VM, Garcia-Godoy F. Endod Dent Traumatol 8: 213-214; 1992.

4. Onetto JE, Flores MT, Garbarino ML. Endod Dent Traumatol 10: 223-227; 1994.

5. Motohiro Kikuchi, Yasukazu Noji, Naoto Higuch, et al. Nition Univ. Dent J 68: 759; 1994.

6. Oikarinen K, Kassila O. Endod Dent Traumatol 3: 172-177; 1987.

7. Naoto Osuga, Masayuki Kasai, Toshio Onishi, et al. Matsumoto Dent J 19: 163; 1993. 